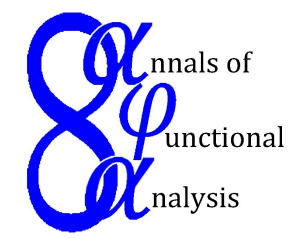

Ann. Funct. Anal. 6 (2015), no. 3, 73-86

http://doi.org/10.15352/afa/06-3-7

ISSN: 2008-8752 (electronic)

http://projecteuclid.org/afa

\title{
CONVEX COMPONENTS AND MULTI-SLICES IN REAL TOPOLOGICAL VECTOR SPACES
}

\author{
F. J. GARCÍA-PACHECO \\ Communicated by B. Ricceri
}

\begin{abstract}
It is shown that, in a non-necessarily Hausdorff real topological vector space, if a subset is a countable disjoint union of convex sets closed in the subset, then those convex sets must be its convex components. On the other hand, by means of convex components we extend the notion of extreme point to non-convex sets, which entails a new equivalent reformulation of the KreinMilman property (involving drops among other objects). Finally, we study the nature of convex functions and provide some results on their support in order to introduce the concept of multi-slice, that is, slices determined by convex functions (instead of by linear functions). Among other things, we prove that the boundary of a closed convex set with non-empty interior can be obtained as the set of support points of a certain lower semi-continuous convex function on that convex set.
\end{abstract}

\section{INTRODUCTION AND PRELIMINARIES}

The theory of (arc-)connected components plays a fundamental role in general topology with numerous applications to other fields of mathematics like geometry or analysis. In this sense, in [13] this trend is followed by introducing the convex components of a non-empty set in a real vector space. As an application of this relatively new theory of convex components we extend the concept of extreme point to non-convex sets, which will allow us to equivalently reformulate the Krein-Milman property. We remind the reader that a Hausdorff locally convex topological vector space is said to have the Krein-Milman property provided that every bounded, closed, and convex subset has extreme points (see [9]).

Date: Received: Dec. 20, 2014; Accepted: Dec. 27, 2014.

2010 Mathematics Subject Classification. Primary 15A03; Secondary 46A55, 46B20.

Key words and phrases. Krein-Milman property, convex set, convex component, extreme point, slice. 
On the other hand, the study of slices has always had a crucial impact on the theory of vector measures. We remind the reader that a slice of a convex set in a real vector space is the intersection of that convex set with the upper half-plane determined by a linear function. Actually, the slices serve as a bridge between vector measures and the geometry of Banach spaces. One of the most representative examples of such connection is given by the Phelps' characterization of the Radon-Nikodym property in terms of strongly exposed points, in other words, a Banach space has the Radon-Nikodym property if and only if every bounded, closed, and convex subset is the closed convex hull of its strongly exposed points (see $[11,3]$ ). As a consequence of this interesting result we have that the Radon-Nikodym property implies the Krein-Milman property (since every strongly exposed point is trivially an extreme point). We remind the reader that a strongly exposed point of a convex set is a point of the boundary of that set on which the set is supported by a continuous linear functional in such a way that every open set containing that point also contains a slice of that set determined by the linear functional. Another application of slices appears in a very recent paper (see [6]) and its aimed, amid other things, at finding a proper equivalent renorming of weakly compactly generated Banach spaces in such a way that the set of non-norm-attaining functionals is nowhere dense.

The final part of this manuscript is devoted to study the algebraic nature of convex functions in order to define the concept of multi-slice, that is, slices determined by convex functions (instead of just by linear functions). We call them multi-slices because in many cases these multi-slices have many convex components (as opposed to slices which typically are convex). As an application of this we show that the boundary of a closed convex set with non-empty interior can be obtained as the set of support points of a convenient lower semi-continuous convex function on that convex set.

\section{Convex components}

This section is devoted to continue the study of the convex components of a non-empty subset of a real vector space already initiated in [13] and successive manuscripts. The reader may observe that there will be some sort of similarity with the theory of connected components of a topological space. However, many properties verified by the connected components are not shared by the convex components.

Definition 2.1 (Valentine, 1964; [13]). Let $X$ be a real vector space. Let $M$ be a non-empty subset of $X$. A subset $C$ of $M$ is said to be a convex component of $M$ if $C$ is a maximal element of the set

$$
\{D \subseteq M: D \text { is convex }\}
$$

partially ordered by the inclusion.

The first result we present here assures that every convex subset of a given set is contained in a convex component. 
Theorem 2.2. Let $X$ be a real vector space. Let $M$ be a non-empty subset of $X$. Every convex subset $C$ of $M$ is contained in a convex component of $M$.

Proof. Consider the non-empty inductive set

$$
\{D \subseteq M: D \text { is convex and } C \subseteq D\}
$$

partially ordered by the inclusion. If $\left(D_{i}\right)_{i \in I}$ is a chain of the previous set, then $C \subseteq \bigcup_{i \in I} D_{i}$ and $\bigcup_{i \in I} D_{i}$ is clearly convex, therefore it is an upper bound for the previous chain. In virtue of the Zorn's Lemma, the previous set has a maximal element. It is not difficult to see that any maximal element of the above set must be a convex component of $M$.

As an immediate consequence of this result we have that the convex components form a covering of the corresponding set. Later we will determine the cases where the convex components form a partition. Now we will show that the convex components of a closed subset of a topological vector space are also closed (this result along with its proof can be found in [13] although we prefer to state it and prove it here for the sake of completeness).

Theorem 2.3. Let $X$ be a real topological vector space. If $M$ is a non-empty closed subset of $X$, then every convex component of $M$ is also closed.

Proof. Let $C$ be a convex component of $M$. It is well known that $\mathrm{cl}(C)$ is convex. On the other hand, $C \subseteq \operatorname{cl}(C) \subseteq M$. Therefore, by the maximality of $C$ we deduce that $C=\operatorname{cl}(C)$.

Observe that this result cannot be generalized to the fact that the convex components are always closed in the corresponding set, as the next example shows.

Example 2.4. Consider in $\mathbb{R}^{2}$ the set

$$
M:=\bigcup_{\ell_{\infty}^{2}} \cup\{(1,1),(1,-1)\},
$$

where $U_{\ell_{\infty}^{2}}$ denotes the open unit ball of $\ell_{\infty}^{2}$. Every point in $U_{\ell_{\infty}^{2}}$ is contained in two different convex components, none of them is closed in $M$.

An immediate consequence of Theorem 2.3 is the fact that in any real vector space if a non-empty set is linearly closed, then its convex components are also linearly closed. We remind the reader that in a real vector space a set is said to be linearly closed provided that its complementary is linearly open, i.e., all of its points are internal points (see [2]).

Corollary 2.5. Let $X$ be a real vector space. If $M$ is a non-empty linearly closed subset of $X$, then every convex component of $M$ is also linearly closed.

Proof. It is sufficient to realize that in any real vector space a set is linearly closed if and only if it is closed when the vector space is endowed with the finest locally convex vector topology.

Notice that the set $M$ in Example 2.4 has two convex components which are not disjoint. This was never the case with the connected components. The 
next observation details in what cases a set is the disjoint union of its convex components.

Fact 2.6. Let $X$ be a real vector space. Let $M$ be a non-empty subset of $X$. The following conditions are equivalent:

(1) For every $m \in M$ there exists only one convex component of $M$ containing $m$.

(2) $M$ is the disjoint union of its convex components.

The final result in this section is a sufficient condition for the convex components to be pairwise disjoint. This sufficient condition involves closedness and countability, so we will strongly rely on Sierpinski's Theorem (see [4, p. 173]), which states that no continuum (compact, connected, and Hausdorff) can be written as a non-trivial countable union of pairwise disjoint closed subsets. However, we first need some preliminary results that we will rely on.

Theorem 2.7 (Armario, García-Pacheco, and Pérez-Fernández, [1]). Let $X$ be a real topological vector space. The set

$$
X_{T}:=\{x \in X: x \text { belongs to any neighborhood of } 0\}
$$

is a closed bounded vector subspace of $X$ whose induced topology is the trivial topology.

Lemma 2.8. Let $X$ be a real topological vector space. Let $a \neq b \in X$. The following conditions are equivalent:

(1) There are a neighborhood of a and a neighborhood of b which are disjoint.

(2) $a-b \notin X_{T}$.

Proof.

$(1) \Rightarrow(2)$ Let $U$ and $V$ be balanced and absorbing neighborhoods of 0 such that $(a+U) \cap(b+V)=\varnothing$. Then $a-b \notin V-U$. It is clear that $V-U$ is a balanced and absorbing neighborhood of 0 . Therefore $a-b \notin X_{T}$.

$(2) \Rightarrow(1)$ By hypothesis there exists a balanced and absorbing neighborhood $V$ of 0 such that $a-b \notin V$. Take another balanced an absorbing neighborhood of 0 such that $U+U \subseteq V$. Finally, $(a+U) \cap(b+U)=\varnothing$.

Lemma 2.9. Let $X$ be a real topological vector space. Let $c \neq d \in X$. Then $[c, d]$ is Hausdorff if and only if $[c, d] \nsubseteq x+X_{T}$ for every $x \in X$.

Proof.

$\Rightarrow$ If $[c, d] \subseteq x+X_{T}$ for some $x \in X$, then $[c, d]$ has the trivial topology in virtue of Theorem 2.7 and thus it cannot be Hausdorff.

$\Leftarrow$ Suppose that $[c, d] \nsubseteq x+X_{T}$ for every $x \in X$. Let $a \neq b \in[c, d]$. Notice that $a-b \notin X_{T}$. Indeed, if $a-b \in X_{T}$, then $[c, d] \subseteq b+X_{T}$ which contradicts the hypothesis. As a consequence, $a-b \notin X_{T}$ and by Lemma 2.8 there are a neighborhood of $a$ and a neighborhood of $b$ which are disjoint. The arbitrariness of $a$ and $b$ shows that $[c, d]$ is Hausdorff. 
Now we are in the right position to prove a sufficient condition for the convex components to be pairwise disjoint.

Theorem 2.10. Let $X$ be a real topological vector space. Let $M$ be a countable disjoint union of convex subsets of $X$ which are closed in $M$. Then the convex components of $M$ are exactly those closed convex sets.

Proof. Let us write

$$
M=\bigcup_{i \in I}^{\cdot} C_{i}
$$

where $I$ is countable and each $C_{i}$ is a non-empty convex subset of $X$ which is closed in $M$. We will follow two steps:

- First, we will show that each $C_{i}$ is a convex component of $M$. So, fix an arbitrary $j \in I$. It is easy to understand, by bearing in mind Theorem 2.2 , that $C_{j}$ is contained in a convex component $D$ of $M$. Assume to the contrary that there exists $d \in D \backslash C_{j}$ and consider any $c \in C_{j}$. Consider the segment $[c, d]$. We will distinguish two possibilities to end up reaching a contradiction:

(1) $[c, d]$ is Hausdorff. In this case, $[c, d]$ is homeomorphic to the closed interval $[0,1]$ and thus it is a continuum. Now we can write

$$
[c, d]=\bigcup_{i \in I}^{\cdot}\left(C_{i} \cap[c, d]\right),
$$

where each $C_{i} \cap[c, d]$ is closed in $[c, d]$ due to the fact that $C_{i}$ is closed in $M$ and $[c, d]$ is contained in $M$. However, this contradicts that $[c, d]$ is a continuum in virtue of Sierpinski's Theorem (see $[4, \mathrm{p}$. $173])$.

(2) $[c, d]$ is not Hausdorff. In this case we have that $[c, d] \subseteq x+X_{T}$ for some $x \in X$ in virtue of Lemma 2.9. As a consequence, $[c, d]$ has the trivial topology in accordance to Theorem 2.7. On the other hand, $C_{j} \cap[c, d]$ is non-empty and closed in $[c, d]$, therefore $C_{j} \cap[c, d]=[c, d]$, which contradicts the fact that $d \notin C_{j}$.

- In the second and final place, we will show that if $D$ is a convex component of $M$, then $D=C_{j}$ for some $j \in I$. There must exist $j \in I$ such that $D \cap C_{j} \neq \varnothing$. If $D \backslash C_{j} \neq \varnothing$, then we can use a similar argument as above to reach a contradiction. As a consequence, $D \subseteq C_{j}$ and by maximality we have that $D=C_{j}$.

The next example shows that the hypotheses of countability and closedness in the previous result are vital.

\section{Example 2.11.}

- Theorem 2.10 does not hold if we eliminate the hypothesis of countability (even keeping the hypothesis of closedness). Indeed,

$$
[0,1]=\bigcup_{x \in[0,1]}\{x\} \text {. }
$$


- Theorem 2.10 does not hold if we eliminate the hypothesis of closedness (even keeping the hypothesis of countability). Indeed, consider in $\mathbb{R}^{2}$ the set

$$
M:=\mathrm{B}_{\ell_{2}^{2}}((-1,0), 1) \cup \mathrm{U}_{\ell_{2}^{2}}((1,0), 1),
$$

where $\mathrm{B}_{\ell_{2}^{2}}$ and $\mathrm{U}_{\ell_{2}^{2}}$ denote the closed and open unit ball of $\ell_{2}^{2}$, respectively. Notice that $M$ is the disjoint union of two convex sets (one of which is not closed in $M)$. However, there are three different convex components of $M$ containing $(0,0): \mathrm{B}_{\ell_{2}^{2}}((-1,0), 1),\{(0,0)\} \cup \mathrm{U}_{\ell_{2}^{2}}((1,0), 1)$, and $[(-2,0),(2,0)]$.

\section{EXTREME POINTS OF NON-CONVEX SETS}

This section is devoted to extend the notion of extreme point to the scope of non-convex sets, which will allow us to equivalently reformulate the KreinMilman property. In this sense, we will generalize a result proven in [1, Theorem 4.1].

We would like to refer the reader to [8] wherein extreme points for non-convex sets are shown to exist if the ambient space has the Radon-Nikodym property.

Definition 3.1. Let $X$ be a real vector space. Let $M$ be a non-empty subset of $X$. An element $m \in M$ is said to be an extreme point of $M$ provided that the following conditions holds: if $C$ is a segment of $M$ containing $m$, then $m$ is an extreme of $C$. The set of extreme points of $M$ is usually denoted by ext $(M)$.

The reader may immediately notice that the notion of extreme point previously defined coincides with the classical notion of extreme point for convex sets. The first result we present here on this topic relates the extreme points of a general non-necessarily convex set with the extreme points of its convex components

Theorem 3.2. Let $X$ be a real vector space. Let $M$ be a non-empty subset of $X$ and let $\left\{C_{i}\right\}_{i \in I}$ be the set of convex components of $X$.

(1) $\operatorname{ext}(M) \subseteq \bigcup_{i \in I} \operatorname{ext}\left(C_{i}\right)$.

(2) If $C_{i} \cap C_{j}=\varnothing$ for all $i \neq j \in I$, then $\operatorname{ext}(M)=\bigcup_{i \in I} \operatorname{ext}\left(C_{i}\right)$.

Proof.

(1) Obvious since $C_{i} \subseteq M$ for every $i \in I$.

(2) Let $x \in \operatorname{ext}\left(C_{i}\right)$ for some $i \in I$. Let $C$ be a segment of $M$ containing $x$. In accordance to Theorem 2.2 there exists $j \in I$ such that $C \subseteq C_{j}$. Therefore $x \in C_{i} \cap C_{j}$ which means by hypothesis that $C_{i}=C_{j}$. Since $x \in \operatorname{ext}\left(C_{i}\right)$, we deduce that $x$ is an extreme of $C$.

Notice that the converse to (2) of Theorem 3.2 does not hold true as shown in the next example.

Example 3.3. Consider the 2-dimensional real Euclidean space, that is, $\ell_{2}^{2}$. Take $M:=2 \mathrm{~B}_{\ell_{2}^{2}} \backslash \mathrm{U}_{\ell_{2}^{2}}$, where $\mathrm{B}_{\ell_{2}^{2}}$ and $\mathrm{U}_{\ell_{2}^{2}}$ denote the closed and open unit ball of $\ell_{2}^{2}$, 
respectively. It is not difficult to observe that the convex components of $M$ are the sets

$$
C_{f}:=M \cap f^{-1}([1,+\infty))
$$

where $f \in \mathrm{S}_{\left(\ell_{2}^{2}\right)^{*}}$. Finally, it can be checked that

$$
\left.\operatorname{ext}(M)=\bigcup_{f \in \mathrm{S}} \operatorname{ext}\left(\ell_{f}^{2}\right)^{*}\right)
$$

However, the convex components of $M$ are not pairwise disjoint.

Our next aim is at providing an equivalent reformulation of the Krein-Milman property. In order for this, we first remind the reader that given a vector space $X$ and a non-empty subset $A$ of $X$, then:

- The balanced hull of $A$ is defined as the intersection of all balanced subsets of $X$ containing $A$ and denoted by bl $(A)$. Furthermore,

$$
\operatorname{bl}(A)=\left\{\lambda a: \lambda \in \mathrm{B}_{\mathbb{K}}, a \in A\right\} .
$$

- The convex hull of $A$ is defined as the intersection of all convex subsets of $X$ containing $A$ and denoted by co $(A)$. Furthermore,

$$
\operatorname{co}(A)=\left\{\sum_{i=1}^{n} t_{i} a_{i}: t_{i} \in[0,1], a_{i} \in A, \sum_{i=1}^{n} t_{i}=1\right\} .
$$

- The absolutely convex hull of $A$ is defined as the intersection of all absolutely convex (convex and balanced) subsets of $X$ containing $A$ and denoted by aco $(A)$. Furthermore,

$$
\operatorname{aco}(A)=\left\{\sum_{i=1}^{n} t_{i} a_{i}: t_{i} \in \mathbb{K}, a_{i} \in A, \sum_{i=1}^{n}\left|t_{i}\right| \leq 1\right\} .
$$

The reader may not forget that the convex hull of a balanced set is absolutely convex but the balanced hull of a convex set may not be convex. On the other hand, we remind the reader about the Krein-Milman property:

Definition 3.4 (Krein and Milman, 1940). Let $X$ be a real topological vector space:

(1) A closed bounded convex subset $M$ of $X$ is said to have the Krein-Milman property exactly when ext $(M) \neq \varnothing$.

(2) $X$ is said to have the Krein-Milmam property exactly when every closed bounded and convex subset of $X$ enjoys the Krein-Milman property.

This definition finds its birth in the very well known Krein-Milmam Theorem (see [9]).

Theorem 3.5 (Krein and Milman, 1940). Let $X$ be a Hausdorff locally convex real topological vector space. Let $M$ be a compact convex subset of $X$. Then $M$ has the Krein-Milman Property. 
The Krein-Milmam property was originally defined for Hausdorff locally convex real topological vector spaces in the following way: A closed bounded convex subset $M$ of a Hausdorff locally convex real topological vector space $X$ is said to have the Krein-Milman property exactly when $M=\overline{\mathrm{co}}(\operatorname{ext}(M))$. Essentially, it can be shown that in a Hausdorff locally convex real topological vector space the fact that every closed bounded convex subset is the closed convex hull of its extreme points is equivalent to the fact that every closed bounded convex subset has an extreme point. However, this equivalence does not hold in general for real topological vector spaces. This is the reason for Definition 3.4. Examples of real topological vector spaces verifying the Krein-Milman property include all Banach spaces with the Radon-Nikodym property (in particular, all reflexive Banach spaces). We will characterize the Krein-Milman property using drops and the balanced hull of convex sets (which, as mentioned earlier, need not be convex). We remind the reader that a drop in a real vector space $X$ is a set of the form co $(M \cup\{x\})$ where $M$ is a convex subset of $X$ and $x \in X \backslash M$. Obviously, it turns out to be that $x \in \operatorname{ext}(\operatorname{co}(M \cup\{x\}))$. We refer the reader to $[12,10,7]$ for a wider perspective on drops in Banach spaces. Our equivalent reformulation starts off with the following lemma:

Lemma 3.6. Let $X$ be a real vector space. Let $M$ be a convex subset of $X$.

(1) If $x \in X \backslash M$, then $\operatorname{ext}(\operatorname{co}(M \cup\{x\})) \backslash\{x\} \subseteq \operatorname{ext}(M)$.

(2) If $0 \notin M$, then $\mathrm{bl}(M)=\operatorname{co}(M \cup\{0\}) \cup \operatorname{co}(-M \cup\{0\})$.

(3) If $0 \notin M$, then $\operatorname{ext}(\operatorname{bl}(M))=(\operatorname{ext}(\operatorname{co}(M \cup\{0\})) \cup \operatorname{ext}(\operatorname{co}(-M \cup\{0\}))) \backslash$ $\{0\}$.

(4) $\operatorname{ext}(\mathrm{bl}(M)) \subseteq \operatorname{ext}(M) \cup \operatorname{ext}(-M)$.

Proof.

(1) Let $t m+(1-t) x \in \operatorname{ext}(\operatorname{co}(M \cup\{x\})) \backslash\{x\}$ where $m \in M$ and $t \in[0,1]$. Observe that $t>0$ since $t m+(1-t) x \neq x$. On the other hand, $t=1$ since $t m+(1-t) x \in \operatorname{ext}(\operatorname{co}(M \cup\{x\}))$.

(2) It suffices to notice that $\operatorname{co}(M \cup\{0\}) \cup \operatorname{co}(-M \cup\{0\})$ is the smallest balanced set containing $M$.

(3) It is a direct consequence of (1) and (2).

(4) It is a direct consequence of (1) and (3).

Now we are in the right position to state and prove the main theorem in this section.

Theorem 3.7. Let $X$ be a Hausdorff locally convex real topological vector space. The following conditions are equivalent:

(1) $X$ has the Krein-Milmam property.

(2) If $M \subseteq X$ is closed, bounded, and convex and $x \in X \backslash M$, then

$$
\operatorname{ext}(\operatorname{co}(M \cup\{x\})) \backslash\{x\} \neq \varnothing .
$$

(3) If $M \subseteq X$ is closed, bounded, and convex and $0 \notin M$, then $\operatorname{ext}(\mathrm{bl}(M)) \neq$ $\varnothing$. 
Proof.

$(1) \Rightarrow(2)$ Assume that $X$ has the Krein-Milmam property. Let $M \subseteq X$ be closed, bounded, and convex and consider $x \in X \backslash M$. Since $X$ is Hausdorff and locally convex and due to the fact that co $(M \cup\{x\})$ is closed, bounded, and convex, we have that

$$
\operatorname{co}(M \cup\{x\})=\overline{\operatorname{co}}(\operatorname{ext}(\operatorname{co}(M \cup\{x\}))) .
$$

If $\operatorname{ext}(\operatorname{co}(M \cup\{x\}))=\{x\}$, then

$$
\operatorname{co}(M \cup\{x\})=\overline{\operatorname{co}}(\operatorname{ext}(\operatorname{co}(M \cup\{x\})))=\{x\},
$$

which is impossible since $x \notin M$. As a consequence,

$$
\operatorname{ext}(\operatorname{co}(M \cup\{x\})) \backslash\{x\} \neq \varnothing .
$$

$(2) \Rightarrow(3)$ Let $M$ be a closed, bounded, and convex subset of $X$ not containing 0 . In accordance to (2) of Lemma 3.6, we have that

$$
\mathrm{bl}(M)=\operatorname{co}(M \cup\{0\}) \cup \operatorname{co}(-M \cup\{0\}) .
$$

By hypothesis,

$$
\operatorname{ext}(\operatorname{co}(M \cup\{0\})) \backslash\{0\} \neq \varnothing .
$$

At this point it only remains to apply (3) of Lemma 3.6.

$(3) \Rightarrow(1)$ Let $M$ be a closed, bounded, and convex subset of $X$. By making a translation if necessary, we can assume without any loss of generality that $0 \notin M$. By hypothesis we have that ext $(\mathrm{bl}(M)) \neq \varnothing$. Finally, in virtue of (4) in Lemma 3.6, we deduce that $\operatorname{ext}(M) \neq \varnothing$.

\section{Multi-Slices Determined By CONVEX FunCtions}

In this final section we will define multi-slices by means of a convex function. We can think of this as follows: linear functions are to slices what convex functions are to multi-slices. The most representative example of a multi-slice is the bi-slice, that is, the multi-slice determined by the absolute value of a linear function. These bi-slices are called like that because they have two convex components (each of them a slice) when the convex set is balanced. Bi-slices appeared for the first time in the literature of Banach spaces in [5] in order to show that if a real Banach space admits an equivalent Féchet smooth norm, then it admits an equivalent renorming in such a way that the set of non-norm-attaining functionals is nowhere dense. Other examples of multi-slices will be given in the further subsections of this section. In particular, we will show that there is a convex function determining a multi-slice with uncountably many convex components. Finally, we will prove that, in Hausdorff locally convex real topological vector spaces, the boundary of a closed convex subset with non-empty interior can be obtained via a multi-slice determined by a convenient lower semi-continuous convex function (this result is the analogue for convex functions of the one that states that every maximal face of a closed convex subset with non-empty interior is an exposed face). 
4.1. Convex functions and their support. We recall the reader that a real valued function $f: C \rightarrow \mathbb{R}$ defined on a convex subset $C$ of a real vector space $X$ is called convex if, for any two points $c_{1}$ and $c_{2}$ in $C$ and any $t \in[0,1]$,

$$
f\left(t c_{1}+(1-t) c_{2}\right) \leq t f\left(c_{1}\right)+(1-t) f\left(c_{2}\right) .
$$

Or, equivalently, the set

$$
\{(c, s) \in C \times \mathbb{R}: f(c) \leq s\}
$$

is convex in $X \times \mathbb{R}$. A function $f$ is said to be concave if $-f$ is convex. Observe that if $f_{1}, \ldots, f_{n}$ are convex functions and $\lambda_{1}, \ldots, \lambda_{n} \geq 0$, then both $\lambda_{1} f_{1}+$ $\cdots+\lambda_{n} f_{n}$ and $\max \left\{f_{1}, \ldots, f_{n}\right\}$ are also convex functions. Our first result in this subsection is a very basic but fundamental theorem which determines what convex sets coincide with the upper part of the graph of a convex function.

Theorem 4.1. Let $X$ be a real vector space. Let $D$ be a convex subset of $X \times \mathbb{R}$. The following conditions are equivalent:

(1) There exist a convex subset $C$ of $X$ and a convex function $f: C \rightarrow \mathbb{R}$ such that

$$
D=\{(c, s) \in C \times \mathbb{R}: f(c) \leq s\} .
$$

(2) The following two assertions hold:

(a) For every $(c, s) \in D$ we have that $\{(c, t) \in X \times \mathbb{R}: s \leq t\} \subseteq D$.

(b) For every $c \in \pi_{X}(D)$ there exists $\min \{s \in \mathbb{R}:(c, s) \in D\}$.

Proof.

$(1) \Rightarrow(2)$ If $(c, s) \in D$ and $t \geq s$, then $t \geq s \geq f(c)$ and by hypothesis $(c, t) \in D$. On the other hand, if $c \in \pi_{X}(D)$, then

$$
f(c)=\min \{s \in \mathbb{R}:(c, s) \in D\} .
$$

$(2) \Rightarrow(1)$ Take $C:=\pi_{X}(D)$ and define the following function:

$$
\begin{aligned}
f: C & \rightarrow \mathbb{R} \\
c & \mapsto f(c):=\min \{s \in \mathbb{R}:(c, s) \in D\} .
\end{aligned}
$$

In order to finish the proof it suffices to show that

$$
D=\{(c, s) \in C \times \mathbb{R}: f(c) \leq s\},
$$

which is immediate by bearing in mind the assertions in (a) and in (b).

The final part of this subsection is aimed at studying the internal structure of the support of convex functions. We begin with the following result, which relies on the concept of "internal point" (see [2]). Given a real vector space $E$ and a non-empty subset $A$ of $E$, we say that $a \in E$ is an internal point of $A$ when every straight line passing through $a$ has a small interval around $a$ entirely contained in $A$. More precisely, $a \in E$ is an internal point of $A$ when, for every $x \in E$, there exists $\delta_{x}>0$ such that $a+\lambda x \in A$ for all $\lambda \in\left[0, \delta_{x}\right]$. The set of internal points of $A$ is denoted by inter $(A)$. Well known facts about internal points, which can be found in [2], include the following: 
- In any topological vector space if a subset $A$ is (absolutely) convex and $\operatorname{inter}(A) \neq \varnothing$, then inter $(A)$ is also (absolutely) convex and $\operatorname{cl}($ inter $(A))=$ $\operatorname{cl}(A)$.

- Every open set of any topological vector space is composed only of internal points.

- The finest locally convex vector topology on a given vector space is the one whose open sets are exactly the convex sets composed only of internal points.

Theorem 4.2. Let $X$ be a real vector space. Let $f: X \rightarrow \mathbb{R}$ be a convex function. If inter $\left(f^{-1}(0)\right) \neq \varnothing$, then $f \geq 0$ and hence $f^{-1}(0)$ is convex.

Proof. Suppose to the contrary that there exists $y \in X$ such that $f(y)<0$. Take any $x \in \operatorname{inter}\left(f^{-1}(0)\right)$. There exists $t>0$ sufficiently small such that $t y+(1-t) x \in f^{-1}(0)$. Now

$$
0=f(t y+(1-t) x) \leq t f(y)<0,
$$

which is a contradiction. Finally, since $f$ is a positive convex function we have that

$$
\operatorname{co}\left(f^{-1}(0)\right) \subseteq f^{-1}((-\infty, 0])=f^{-1}(0)
$$

4.2. Slices, bi-slices, and multi-slices. Recall that a slice of a convex subset $M$ of a real vector space $X$ is a set of the form

$$
\operatorname{slc}(M, f, \delta):=f^{-1}([\delta,+\infty)) \cap M,
$$

where $f: X \rightarrow \mathbb{R}$ is a linear function and $\delta$ is a real number. The reader may notice that slc $(M, f, \delta)$ is always a convex set as long as it is non-empty. However, sometimes in the literature of Banach spaces and topological vector spaces slices determined by the absolute value of linear functions are also considered. We call bi-slices to this type of slices. Trivially the absolute value of a linear function is not linear but convex. This motivates us to introduce the following definition.

Definition 4.3. Let $X$ be a real vector space. Let $M$ be a convex subset of $X$. The multi-slice of $M$ determined by a convex function $f: X \rightarrow \mathbb{R}$ and a real number $\delta$ is defined by

$$
\operatorname{slc}(M, f, \delta):=f^{-1}([\delta,+\infty)) \cap M .
$$

Our aim in this section is at determining the convex components of multi-slices. We will begin by the bi-slices.

Theorem 4.4. Let $X$ be a real vector space. Let $M$ be a convex subset of $X$. Consider a linear function $f: X \rightarrow \mathbb{R}$ and a real number $\delta$.

(1) If $\delta \leq 0$, then $\operatorname{slc}(M,|f|, \delta)=M$ and thus it has only one convex component.

(2) If $\delta>0$, then

$$
\operatorname{slc}(M,|f|, \delta)=\operatorname{slc}(M, f, \delta) \dot{\cup} \operatorname{slc}(M,-f, \delta),
$$

and thus it has at most two convex components. 
Proof. If $\delta \leq 0$, then there is really nothing to prove so let us suppose that $\delta>0$. Now observe that if $X$ is endowed with the finest locally convex vector topology, then $f$ is continuous and thus slc $(M, f, \delta)$ and slc $(M,-f, \delta)$ are both closed in $M$. Finally, Theorem 2.10 assures that $\operatorname{slc}(M, f, \delta)$ and $\operatorname{slc}(M,-f, \delta)$ are the only convex components of slc $(M, f, \delta)$ (provided they are non-empty).

The next result shows why bi-slices are called like that.

Theorem 4.5. Let $X$ be a real vector space. Let $M$ be a convex subset of $X$ with $\operatorname{inter}(M) \neq \varnothing$. The following conditions are equivalent:

(1) $M$ is absolutely convex.

(2) If $f: X \rightarrow \mathbb{R}$ is linear, $\delta>0$, and $\operatorname{slc}(M,|f|, \delta) \neq \varnothing$, then $\operatorname{slc}(M,|f|, \delta)$ has exactly two convex components.

Proof.

$(1) \Rightarrow(2)$ Let $m \in \operatorname{slc}(M,|f|, \delta)$. Note that we may assume without loss of generality that $m \in \operatorname{slc}(M, f, \delta)$. Since $M$ is symmetric it is easy to see that $-m \in \operatorname{slc}(M,-f, \delta)$. By $(2)$ in Theorem 4.4 we have that $\operatorname{slc}(M,|f|, \delta)$ has two convex components.

$(2) \Rightarrow(1)$ Suppose to the contrary that $M$ is not symmetric. There exists $m \in M$ such that $-m \notin M$. Now consider $X$ endowed with the finest locally convex vector topology. With this topology $X$ turns out to be Hausdorff and locally convex, so the Hahn-Banach Separation Theorem allows us to deduce the existence of an element $f \in X^{*}$ such that

$$
1=f(-m)>\sup f(M) .
$$

Finally observe that $\operatorname{slc}(M, f, 1)=\varnothing$ whereas slc $(M,-f, 1) \neq \varnothing$, therefore $\operatorname{slc}(M,|f|, 1)$ is convex and thus it only has one convex component.

Remark 4.6. Let $X$ be a real vector space. Let $M$ be a convex subset of $X$.

- Consider a linear function $f: X \rightarrow \mathbb{R}$ and a real number $\delta$. We can now form a new convex function $g:=\lambda f^{+}+\gamma f^{-}$with $\lambda, \gamma>0$.

(1) If $\delta \leq 0$, then $\operatorname{slc}(M, g, \delta)=M$ and thus it has only one convex component.

(2) If $\delta>0$, then

$$
\operatorname{slc}(M, g, \delta)=\operatorname{slc}\left(M, f, \frac{\delta}{\lambda}\right) \dot{\cup} \operatorname{slc}\left(M,-f, \frac{\delta}{\gamma}\right),
$$

and thus it has at most two convex components.

- More generally, let $f_{1}, \ldots, f_{n}: X \rightarrow \mathbb{R}$ be positive convex functions with disjoint support and $\lambda_{1}, \ldots, \lambda_{n}, \delta>0$. Then

$$
\operatorname{slc}\left(M, \lambda_{1} f_{1}+\cdots+\lambda_{n} f_{n}, \delta\right)=\bigcup_{i=1}^{n} \operatorname{slc}\left(M, f_{i}, \frac{\delta}{\lambda_{i}}\right),
$$

and thus it has at most $n$ convex components. 


\subsection{A convex function determining a connected multi-slice with un-} countably many convex components. Consider the convex function

$$
\begin{aligned}
f: \mathbb{R}^{2} & \rightarrow \mathbb{R} \\
(x, y) & \mapsto f(x, y):=x^{2}+y^{2}
\end{aligned}
$$

and the convex set $M:=\mathrm{B}_{\ell_{2}^{2}}$. For every $0<\delta<1$, the multi-slice

$$
\operatorname{slc}(M, f, \delta)=M \backslash \delta \mathrm{U}_{\ell_{2}^{2}}
$$

is a connected set with uncountably many convex components.

4.4. Multi-slices which determine the boundary of a closed convex set with non-empty interior. In the theory of Banach spaces or (Hausdorff locally convex) topological vector spaces it is well known that any maximal convex subset of the boundary of a closed convex set with non-empty interior is supported on the convex set by a continuous linear functional. The analogue to this statement for convex functions is the following result.

Theorem 4.7. Let $X$ be a Hausdorff locally convex real topological vector space. Let $M$ be a non-empty closed convex subset of $X$ with non-empty interior. There exists a lower semi-continuous convex function $f: X \rightarrow \mathbb{R}$ and a number $\delta>0$ such that $\operatorname{slc}(M, f, \delta)=\mathrm{bd}(M)$.

Proof. By making a translation if necessary we may assume without any loss of generality that $0 \in \operatorname{int}(M)$. In accordance to the Hahn-Banach Theorem, for every $m$ in the boundary of $M$ we can find a continuous linear functional $m^{*}: X \rightarrow \mathbb{R}$ such that

$$
1=m^{*}(m)=\sup m^{*}(M) .
$$

Define

$$
\begin{aligned}
f: X & \rightarrow \mathbb{R} \\
x & \mapsto f(x):=\sup \left\{m^{*}(x): m \in M\right\} .
\end{aligned}
$$

Notice the following:

- $f$ is well defined. Indeed, it is a direct consequence of the fact that $M$ is absorbing (because 0 is an interior point of $M$ ).

- $f$ is convex. Indeed, it is a direct consequence of the fact that $m^{*}$ is convex for every $m \in M$.

- $f$ is lower semi-continuous. Indeed, it is a direct consequence of the fact that $m^{*}$ is lower semi-continuous for every $m \in M$.

- $\operatorname{slc}(M, f, 1)=\mathrm{bd}(M)$. Indeed, it is a direct consequence of the definition of $f$.

\section{REFERENCES}

1. R. Armario, F.J. García-Pacheco and F.J. Pérez-Férnandez, On the Krein-Milman Property and the Bade Property, Linear Alg. Appl. 436 (2012), no. 5, 1489-1502.

2. N. Bourbaki, Topological vector spaces. Chapters 1-5, Translated from the French by H. G. Eggleston and S. Madan, Elements of Mathematics (Berlin), Springer-Verlag, Berlin, 1987. 
3. V.P. Fonf, J. Lindenstrauss and R.R. Phelps, Infinite dimensional convexity, Handbook of the geometry of Banach spaces, Vol. I, 599-670, North-Holland, Amsterdam, 2001.

4. K. Kuratowski, Topology. Vol. II., New edition, revised and augmented. Translated from the French by A. Kirkor Academic Press, New York-London; Pastwowe Wydawnictwo Naukowe Polish Scientific Publishers, Warsaw 1968.

5. F.J. García-Pacheco, Four Non-Linear Problems on Normed Spaces. Volumen I, Verlag Dr. Müller, Berlin, 2008.

6. F.J. García-Pacheco, The Universal Renorming, Israel J. Math. 202 (2014), no. 1, 405-422.

7. F.J. García-Pacheco and J.B. Seoane-Sepúlveda, Rotundity, smoothness and drops in Banach spaces, Bull. Belg. Math. Soc. Simon Stevin 15 (2008), no.3, 473-478.

8. R.E. Huff and P.D. Morris, Geometric characterizations of the Radon-Nikodm property in Banach spaces., Studia Math. 56 (1976), no. 2, 157-164.

9. M. Krein and D. Milman, On extreme points of regular convex sets, Studia Math. 9 (1940), $133-138$.

10. V. Montesinos, Drop property equals reflexivity, Studia Math. 87 (1987), no. 1, 93-100.

11. R.R. Phelps, Dentability and extreme points in Banach spaces, J. Funct. Anal. 17 (1974), 79-80.

12. S. Rolewicz, On drop property, Studia Math. 85 (1987), no. 1, 27-35.

13. F.A. Valentine, Convex sets, McGraw-Hill, New York, 1964.

Department of Mathematics, University of Cadiz, Puerto Real 11510, Spain.

E-mail address: garcia.pacheco@uca.es 